\title{
RELIABILITY ANALYSIS OF NUMERICAL SIMULATION IN NEAR FIELD BEHAVIOR
}

\author{
Akira KOBAYASHI ${ }^{1}$, Kiyohito YAMAMOTO ${ }^{2}$, Masakazu CHIJIMATSU ${ }^{3}$ \\ and Tomoo FUJITA ${ }^{4}$ \\ ${ }^{1}$ Member of JSCE, Associate Professor, Graduate School of Agricultural Science, Kyoto University \\ (Kitashirakawa-oiwake-cho, Sakyo-ku, Kyoto 606-8502, Japan) \\ E-mail:kobadesu@kais.kyoto-u.ac.jp \\ ${ }^{2}$ Member of JSCE, Assistant, Graduate School of Agricultural Science, Kyoto University \\ (Kitashirakawa-oiwake-cho, Sakyo-ku, Kyoto 606-8502, Japan) \\ E-mail:yama3@kais.kyoto-u.ac.jp \\ 3 Member of JSCE, HAZAMA Corporation \\ (2-2-5, Toranomon, Minato-ku, Tokyo 105-8479, Japan) \\ E-mail:mcjiji@hazama.co.jp \\ ${ }^{4}$ Member of JSCE, Japan Atomic Energy Agency \\ (4-33, Tokai-mura Muramatsu, Naka-gun, Ibaraki, Japan) \\ E-mail: fujita.tomoo@jaea.go.jp
}

\begin{abstract}
The uncertainties of the boundary conditions, the elastic modulus and Poisson's ratio on the mechanical behavior at near field of high level radioactive waste repository were examined. The method used to examine the error propagation was the first order second moment method. The reliability of the maximum principal stress, maximum shear stress at crown of the tunnel and the minimum principal stress at spring line was examined for one million years. For elastic model, the reliability of the maximum shear stress gradually decreased while that of the maximum principle stress increased. That of the minimum principal stress was relatively low for one million years. This tendency was similar to that from the damage model.
\end{abstract}

Key Words : high level radioactive waste disposal, uncertainty analysis, near field, mechanical behavior

\section{INTRODUCTION}

To safely dispose of the high level radioactive waste (HLW), the repository is composed by multi -barrier system. In Japan, the waste is planed to be surrounded by the highly compacted bentonite as a buffer material. The repository is planned to be set at the deep geology under $300 \mathrm{~m}$ from the ground level ${ }^{1)}$. The waste will be isolated from the human activity for very long time. The safety of the repository has to be ensured for a long period.

The geological environment may change for a long period. The average displacement velocity of active faults in Japan is considered to be in a range of about $0.01-10$ meters/1000years ${ }^{2}$. Moreover, the uplift and denudation are the gradual processes that generally extend over an extremely long period. The uplift speed in the Quaternary period is considered to be from $0.5 \mathrm{~mm} /$ year to $1.0 \mathrm{~mm} /$ year for most regions of Japan ${ }^{3)}$. The cycle of cold and warm period, which affects the sea level change, is about
100,000 years $^{4}$. In addition to these kinds of large and long scale change, Japan has the various types of rocks which are distributed irregularly. Therefore, the change in geological environment may be very specific locally, which will cause the uncertainty in the prediction of the safety of the repository.

While the above discussion is related to the geological condition, the near field of the repository has the other uncertainty related to the repository design. The waste generates a heat for a long period and the groundwater level recovers after closing the repository. The phenomena of the far field have an effect on the boundary conditions of the near field. The stability of the ground around the repository tunnel will be affected by the change in the boundary conditions. To discuss the stability of excavation disturbed zone (EDZ), the international cooperative study, DECOVALEX (DEmonstration of COupled models and their VAlidation against EXperiments) - THMC project takes place to support the development of mathematical models of the coupled thermal, hy- 
draulic, mechanical and chemical processes in geological media ${ }^{5}$.

In the task of the project, the effect of chemically degraded properties on the stability of rock around the repository tunnel was discussed. Among possible various chemical phenomena, the effect of sea water was focused on. This is because a few repositories in the world are planed to be built at the coastal area at present. In the task, the examination of uncertainty was also one of the subjects. In this paper, the results examined for the subject are presented.

As mentioned earlier, while there are many uncertainties related to the stability of the repository tunnel, the effects of the change in boundary conditions of near field on the behavior of EDZ are focused on in this study. Uncertainties related to the simulation of ground behaviors arise from two sources: the scatter of properties, and the systematic error in properties and problem establishment. The latter contributes to the numerical evaluation differently from the former because the spatial variability of the parameters averages over the regional volume of ground while the systematic error is constant. Therefore, the systematic error propagates almost unchanged through the analysis and can have a greater influence on predictive uncertainty than does spatial variability ${ }^{6}$. The systematic error consists of errors of the sampling process, bias in the measurement, estimation of boundary conditions and so on. However, the study about the propagation of systematic error has not been carried out for HLW project. On the other hand, the uncertainty induced from the scatter of properties has been examined so much. In particular, the effect of heterogeneity of permeability on the safety assessment has been discussed well ${ }^{7}$. Moreover, the upscaling method of the permeability from small to large region has been studied well ${ }^{8)}$. This is related to the uncertainty of the model of the distribution of the parameters used in the numerical calculation. In this study, the systematic error of boundary conditions at the near field of HLW repository is examined by using the reliability analysis. This is probably the first approach to examine the systematic error for HLW project.

The boundary conditions of the near field change for a very long period. In this study, the boundary conditions for one million years are estimated from the results of simulation of far field. The variances of the boundary conditions are estimated from variation for one million years. This procedure may give a larger estimation of the variance than the case that only the effect of the different waste composition is considered. In addition to the influence of the boundary conditions on the uncertainty of the mechanical behavior, the effect of the fundamental mechanical properties, the elastic modulus and
Poisson's ratio, is also examined. Moreover, the difference of uncertainties due to the different analysis model is also discussed by comparing the results from the elastic model and the damage expansion model.

\section{VARIANCE AND CORRELATION OF VARIABLES}

\section{(1) Boundary conditions of near field}

The boundary condition of near field was obtained from the results of far field model ${ }^{9}$. The far field model had a vertical depth of $1000 \mathrm{~m}$ and horizontal length of $35 \mathrm{~m}$. The repository tunnel was excavated at the depth of $500 \mathrm{~m}$. As a rock, a crystalline rock such as granite is assumed. The initial horizontal stress was given as $\sigma_{h}=0.055 d+4.6 \mathrm{MPa}$, and the initial vertical stress was $\sigma_{v}=26487 d \mathrm{~Pa}$, where $d$ was the depth from the ground surface with a unit of meter. The vertical thermal gradient was $30^{\circ} \mathrm{C} / \mathrm{km}$ with a fixed temperature of $10^{\circ} \mathrm{C}$ at the ground surface. The groundwater table was fixed at the ground level and the fluid pressure at the bottom boundary was set at $9 \mathrm{MPa}$. Under the above conditions, the repository tunnel was excavated in one-step. After excavation, the waste and the bentonite buffer were installed instantaneously. Postclosure simulation was carried out by considering the heat generation from the waste and the constant swelling pressure of buffer material. Thermal, hydraulic and mechanical behavior of surrounding rock mass was calculated for one million years. Fig. 1 shows the obtained boundary conditions of near field from the analysis of far field. In this study, the variation of boundary conditions for one million years is considered.

\section{(2) Variance and correlation}

The boundary conditions are considered as a variable for the examination of uncertainties of the behavior of near field. Fig.2 shows the model of near field. The size of vertical boundary is the same as that of the horizontal one. The vertical, the horizontal and the radial stresses obtained from the far field examination are used as the boundary conditions, and the temperature and the fluid pressure are applied to the entire region of near field model as an interior condition, meaning that the transfer of heat and fluid pressure is not considered in the near field model. The effect of these variables on the behavior of near field is examined in this study.

The correlation coefficients of five variables: the inner conditions of temperature $T$ and fluid pressure $P$, and the boundary conditions of vertical stress $\sigma_{v}$, 
Table 2 Standard deviation and increment of each variable

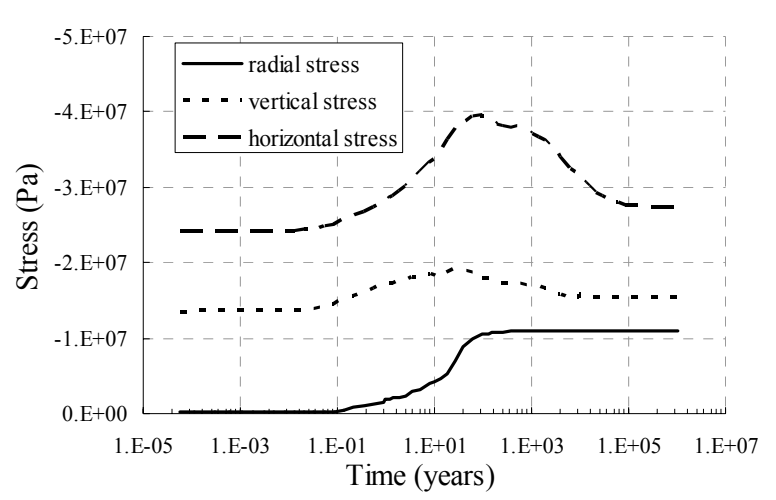

(a) Stress evolution

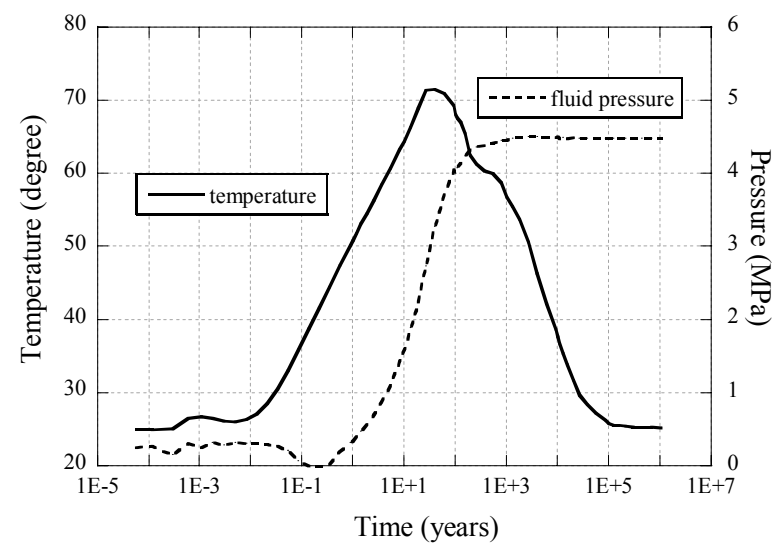

(b) Fluid pressure and temperature evolution Fig. 1 Conditions of near field model

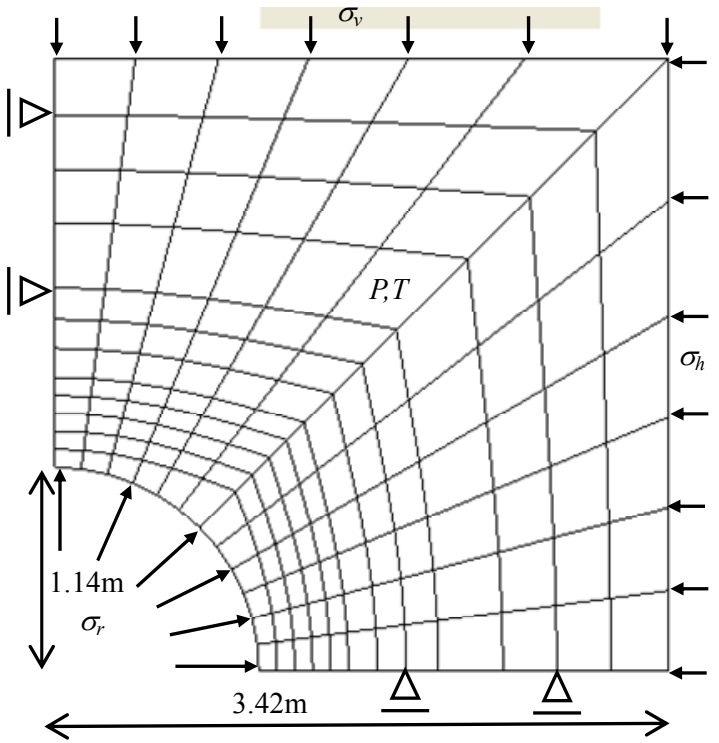

Fig.2 Model of near field analysis

$\sigma_{h}$ : Horizontal stress, $\sigma_{v}$ : Vertical stress, $\sigma_{r}$ : Radial stress, $P$ : Fluid pressure, $T$ : Temperature.

Table 1 Correlation coefficient of boundary conditions

\begin{tabular}{ccccc}
\hline & $\sigma_{v}$ & $\sigma_{r}$ & $P$ & $T$ \\
\hline$\sigma_{h}$ & 0.829 & 0.629 & -0.590 & -0.870 \\
$\sigma_{v}$ & - & 0.347 & -0.280 & -0.930 \\
$\sigma_{r}$ & - & - & -0.996 & -0.21 \\
$P$ & - & - & - & 0.15 \\
\hline
\end{tabular}

\begin{tabular}{ccccc}
\hline & $\sigma_{h}(\mathrm{MPa})$ & $\sigma_{v}(\mathrm{MPa})$ & $\sigma_{r}(\mathrm{MPa})$ & $P(\mathrm{MPa})$ \\
\hline $\mathrm{SD}$ & 5.2 & 1.7 & 4.8 & 2.0 \\
$\Delta x$ & 1 & 1 & 0.02 & 0.01 \\
\hline & $T\left({ }^{\circ} \mathrm{C}\right)$ & $E(\mathrm{GPa})$ & $v$ & \\
\hline $\mathrm{SD}$ & 16 & 21 & 0.03 & \\
$\Delta x$ & 3 & 0.1 & 0.001 &
\end{tabular}

SD: Standard deviation, $\Delta x$ : increment

Table 3 Elastic parameters used for examination

\begin{tabular}{ll}
\hline Parameter & Value \\
\hline Elastic Modulus & $70 \mathrm{GPa}$ \\
Poisson's ratio & 0.3 \\
Thermal expansion coefficient & $1 \cdot 10^{-5}{ }^{\circ} \mathrm{C}^{-1}$ \\
\hline
\end{tabular}

horizontal stress $\sigma_{h}$ and radial stress $\sigma_{r}$, are calculated from the temporal change shown in Fig.1. The result is shown in Table 1. It is found that temperature has a high correlation with the horizontal and the vertical stresses, and the fluid pressure has high correlation with the radial stress.

While the variance of the stress boundary conditions has to be evaluated from the past measured results of in-situ stress, the measurement giving the information on the variance of in-situ stress have not been obtained. However, since the boundary condition will vary with time during the evaluation period as shown in Fig.1, the variance of the boundary conditions is estimated from the temporal variation for simplicity. Table 2 shows the standard deviation of each variable. As shown in the table, the standard deviation of each variable is relatively high and there is a wide variation among variables. Even if the case that the different wastes are stored in the repository is examined, the relation among the variables from temporal change would be appeared similarly. In the table, the standard deviation of elastic modulus and Poisson's ratio is also indicated. For the examination of the uncertainty of the mechanical behavior of near field, the effect of two parameters is evaluated. The standard deviation of elastic modulus is estimated to be $30 \%$ of the average by referring the result of Lee, et al. ${ }^{10)}$. While that of Poisson's ratio has not been known well, the standard deviation is assumed to be $10 \%$ of the average. Table 3 shows the mean elastic parameters used for examination. Since the effect of thermal expansion coefficient is the same as that of elastic modulus in this problem, the effect of the thermal expansion coefficient is not examined in this study.

In Table 2, the increment of each variable is also indicated. The increment is used to evaluate the partial derivatives by numerical differentiation. The increment of radial stress and fluid pressure is small 
in comparison with that of horizontal and vertical stresses. This is because the fluid pressure and the radial stress are very small at the beginning as shown in Fig. 1. The increment of elastic modulus and Poisson's ratio is set at a small value to reduce the error of partial derivatives.

Although the comparison with the damage expansion model is carried out in this study, the effect of damage parameters on the results is not examined. This is because the resultant variance increases with increase of the number of variables. The damage parameters are assumed to be correct. The effect of seven variables, vertical, horizontal and radial stresses on the boundary, temperature and fluid pressure in the region, elastic modulus and Poisson's ratio, is examined to evaluate the uncertainty of the mechanical behavior at the near field for one million years.

\section{FIRST ORDER SECOND MOMENT METHOD}

There are some methods to examine the reliability index and the probability of event such as the point estimate method ${ }^{11)}$, Hasofer-Lind mehod $^{12)}$ and Monte Carlo simulation. In this paper, the first order second moment method is applied. This is because this method can examine the effect of each variable. Christian, et al. ${ }^{13)}$ applied this method to examine the stability of the dikes.

Examination of uncertainty is to evaluate the error propagation of each variable. The error of the variables propagates through the calculation and affects the final result. The result of the calculations can be considered to be a function $g$ of the several variables. The variables $\left(X_{1}, X_{2}, \ldots, X_{n}\right)$ are evaluated at some values $\left(x_{1}, x_{2}, \ldots, x_{n}\right)$. Thus,

$$
g=g\left(x_{1}, x_{1}, \cdots, x_{n}\right)
$$

The first order of Taylor's series for multiple variables gives

$$
\begin{aligned}
g\left(x_{1}, x_{1}, \cdots, x_{n}\right) \approx & g\left(\mu_{X 1}, \mu_{X 2}, \cdots, \mu_{X n}\right) \\
& +\frac{1}{1 !} \sum_{i=1}^{n}\left(x_{i}-\mu_{X i}\right) \frac{\partial g}{\partial x_{i}}
\end{aligned}
$$

The partial derivatives are taken at $\mu_{X 1}, \mu_{X 2}, \ldots$, $\mu_{X n} . \mu_{X i}$ is the mean value of $X_{i}$. The expected value of $g$ is assumed to be given by the value of the function calculated with the mean values of all the variables.

$$
\mu_{g} \approx g\left(\mu_{X 1}, \mu_{X 2}, \cdots, \mu_{X n}\right)
$$

The variance of a function $g$ is given from Equations (2) and (3) by

$$
\begin{aligned}
\sigma_{g}^{2} & =E\left[\left(g-\mu_{g}\right)^{2}\right] \approx E\left[\left(\sum_{i=1}^{n}\left(x_{i}-\mu_{X i}\right) \frac{\partial g}{\partial x_{i}}\right)^{2}\right] \\
& =\sum_{i=1}^{n} \sum_{j=1}^{n} \rho_{X i} \sigma_{X j} \sigma_{X j} \frac{\partial g}{\partial x_{i}} \frac{\partial g}{\partial x_{j}} \\
& =\sum_{i=1}^{n} \sigma_{X i}^{2}\left(\frac{\partial g}{\partial x_{i}}\right)^{2}+\sum_{i=1}^{n} \sum_{j \neq i}^{n} \operatorname{Cov}\left(X_{i}, X_{j}\right) \frac{\partial g}{\partial x_{i}} \frac{\partial g}{\partial x_{j}}
\end{aligned}
$$

The approximation sign replaces the equal sign because the higher order terms of Taylor's series are assumed to be small. The partial derivative in Equation (4) is calculated numerically by

$$
\frac{\partial g}{\partial x_{i}} \approx \frac{\Delta g}{\Delta x_{i}}
$$

While the central difference needs to increase and decrease the variable by a small amount, the differentiation is carried out by increasing the variable from the mean value. This is because the numerical efficiency is not good since each numerical calculation has to be carried out for one million years. The increment $\Delta x$ used for each variable is shown in Table 2. The correlation coefficient among each variable is indicated in Table $\mathbf{1}$ and the variance is obtained from the standard deviation in Table 2. Fig. 3 shows the incremented horizontal stress. In this case, $1 \mathrm{MPa}$ is added to the value obtained from the far field analysis for one million years. The evolution of boundary conditions from far field analysis is considered as a mean value of boundary conditions. In similar way, the vertical and radial stresses, temperature and fluid pressure are incremented. While the increment of each variable has been suggested to be equal to the standard deviation, the value is set to be smaller than the standard deviation to reduce the error of the numerical differentiation. While the confirmation of the estimation error of the partial derivative is not carried out for the low numerical efficiency, the estimation error is expected to be small for the relatively small increment. In particular, since the increments of the elastic properties are set to be very small, the estimation error will be small. For the boundary conditions, while the increments of the horizontal and vertical stresses are relatively large, the effect of the increment of $1 \mathrm{MPa}$ on the behavior of crystalline rock having the elastic properties shown in Table 3 is expected to be very small. Even if the behavior is nonlinear, the difference of the occurrence of the nonlinearity by the increment of 1 


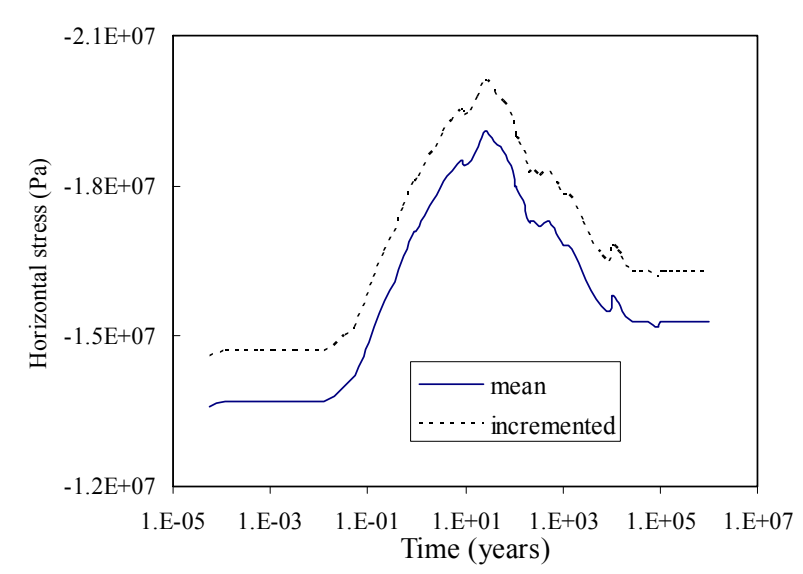

Fig. 3 Incremented boundary condition of horizontal stress

MPa can be considered to be negligible. Contribution of variable $X_{i}$ to the variance $\sigma_{g}^{2}$ is given by

$$
\sigma_{X i}^{2}\left(\frac{\partial g}{\partial x_{i}}\right)^{2}+\sum_{j \neq i}^{n} \operatorname{Cov}\left(X_{i}, X_{j}\right) \frac{\partial g}{\partial x_{i}} \frac{\partial g}{\partial x_{j}}
$$

If this contribution is large, it can be understood that the variable $X_{i}$ has a large uncertainty for the result $g$. The reliability index of function $g$ is given by

$$
\beta=\mu_{g} / \sigma_{g}
$$

When the reliability index is high, it can be understood that the uncertainty of the function $g$ is low.

In this study, the behavior of near field of HLW repository is examined. Although the function $g$ is usually the safety factor in the geotechnical problem, the safety factor of the ground around the repository tunnel is not a problem after closing the repository. It is more worried that the mechanical behavior of the ground causes a bad effect on the function to prevent the contaminant transport. As a function $g$, the maximum principal stress at the crown of the tunnel, the minimum principal stress at the spring line and the maximum shear stress at the crown are examined. This is because each stress has the highest value at the location in the elastic model. Moreover, the damage variable and the permeability at the crown, which are estimated from the damage analysis, are also examined. The difference from the result obtained by using the mean boundary conditions and mechanical properties is considered as $\Delta g$, which value is divided by the $\Delta x$ shown in Table 2 to obtain the partial derivative. In the examination of HLW disposal project, the examination period is very long. The boundary and interior conditions also change through a long period as shown in Fig.1. Therefore, the contribution of each variable to the mechanical behavior may change temporally. To investigate the temporal change of contribution of each variable, the function $g$ at 100, 1,000, 10,000, 100,000, 1,000,000 years after closing the repository is examined. Although the error of numerical differentiation may change through an evaluation period, that is assumed not to affect the examination so much.

\section{NEAR FIELD MODEL}

\section{(1) Elastic model}

To examine the fundamental effect of the uncertainty of each variable, the elastic model is applied. As mentioned earlier, the vertical, horizontal and radial stresses are used as a boundary condition, and the temperature and fluid pressure are applied to the entire region of near field model as an interior condition. In the calculation, the linear thermal expansion and effective stress are incorporated. While the result of model shown in Fig.2 is difficult to compare with the theoretical one, the numerical approach was validated for the quadrilateral model by comparing with the theoretical displacement ${ }^{14)}$.

\section{(2) Damage expansion model}

In damage mechanics, the change in mechanical behavior due to the growth of damage (cracks) in material is considered ${ }^{15}$. This behavior is expressed by means of damage variable $D$. The damage expansion model is based on the damage mechanics theory ${ }^{16}$. For the isotropic damage evolution, the total stress, $\sigma_{i j}$ - strain, $\varepsilon_{i j}$ relation of damage expansion model is expressed by

$$
\begin{aligned}
\sigma_{i j} & =(1-D)\left\{\left(\lambda \varepsilon_{k k} \delta_{i j}+2 \mu \varepsilon_{i j}\right)-\frac{1}{3} \varepsilon_{k k}^{v}(3 \lambda+2 \mu) \delta_{i j}\right\} \\
& -\delta_{i j} P-(3 \lambda+2 \mu) \delta_{i j} \alpha \Delta T
\end{aligned}
$$

where $D$ is the damage variable, $\lambda$ and $\mu$ are Lamé's constants. $\varepsilon_{k k}^{y}$ is the volumetric strain induced by damage development. $P$ is the fluid pressure, $\alpha$ is the linear thermal expansion coefficient, $\delta_{i j}$ is the Kronecker's delta, and $\Delta T$ is the increment of temperature. The damage potential $Y_{e q}$ in relation to $D$ is described as

$$
Y_{e q}=K_{d} D^{n_{d}}+B_{0}
$$

where $K_{d}$ and $n_{d}$ are damage parameters, $B_{0}$ is the initial damage potential. $Y_{e q}$ in relation to $\varepsilon_{k k}^{y}$ is also assumed to be 


$$
Y_{e q}=K_{v}\left(\frac{1}{3} \varepsilon_{k k}^{v}\right)^{n_{v}}
$$

$K_{d}, n_{d}, B_{0}, K_{v}$ and $n_{v}$ are called the damage parameters. The damage potential $Y_{e q}$ is equivalent to the elastic work. When $Y_{e q}$ is larger than $B_{0}$, the damage variable increases ${ }^{17)}$. Then, $B_{0}$ is updated to $Y_{e q} . B_{0}$ becomes the previous maximum damage potential. Therefore, the damage progresses when the elastic work is larger than the past maximum elastic work. It was confirmed that the relation of stress and axial strain and that of stress and volumetric strain at the uniaxial compression test were realized well by the damage expansion model with the identified damage parameters ${ }^{18)}$. In this study, the damage parameters are assumed to be correct. The uncertainty of elastic work induced from the error of elastic properties and boundary conditions will affect the damage development.

By using the volumetric strain due to damage development $\varepsilon_{k k}^{v}$, the change in permeability can be modeled. Now, let $\Delta e_{f}$ be the average aperture increment of a single fracture due to damage development. This can be estimated by using the fracture density $f$ and average fracture length $L$ as

$$
\Delta e_{f}=\frac{4 \varepsilon_{k k}^{v}}{f \cdot L^{2} \pi}
$$

in which the shape of fracture is assumed to be a circle. Using the increment of the aperture of a single fracture, the equivalent permeability $K_{e q}$ is written as following with the cubic law:

$$
K_{e q}=\frac{\rho g_{a}\left(e_{0}+\Delta e_{f}\right)^{3}}{12 \mu_{d}} \cdot f
$$

where $\mu_{d}$ is the dynamic viscosity, $\rho$ is the density of water, $g$ is the gravitational acceleration and $e_{0}$ is the initial aperture. The change in permeability due to damage development is estimated by the above equivalent permeability.

The damage parameters were obtained from the degraded rock sample submerged in saline water for 90 days ${ }^{19}$. The tests were conducted on Ävrö granite from the Äspö Hard Rock Laboratory in Sweden. The damage parameters were quite objectively determined by the test results ${ }^{20)} . Y_{e q}$ was obtained by incorporating the unconfined compression condition. The damage variable $D$ was estimated by the difference of stress from the stress- elastic axial strain relation. The expansive strain due to damage progress was also estimated by the difference of volu- metric strain from the elastic volumetric strain. Then, by using the Equations (9) and (10), $K_{d}, n_{d}, K_{v}$ and $n_{v}$ were determined by the fitting process. The obtained values were $K_{d}=1.7 \mathrm{MPa}, K_{v}=106 \mathrm{MPa}, n_{d}=0.8$ and $n_{v}=0.95$. The fracture density and length used for the examination are given in Table 4.

\section{EXAMINATION RESULTS}

\section{(1) Elastic model}

Table 5 shows the maximum principal stress at crown of the elastic case. In the table, 'Mean' is the case using the mean values for all variables. The column of variable indicates the incremented variable. When one variable is incremented, the other variables are set at the mean value. Similarly, Tables 6 and 7 indicate the minimum principal stress at spring line and maximum shear stress at crown, respectively. As shown in the tables, the method using in this examination does not have an efficiency to examine the effect of temperature. The results are the same as the ones from mean variables. This is because the increment of temperature between time steps is the same as the mean case. Moreover, it is found that the effect of elastic modulus and Poisson's ratio appears for the maximum and the minimum principal stresses until 10,000 years so much. This is because the variation of the elastic parameters affects the thermal stress.

By using the results, the contribution of each variable to the variance is calculated by Equation (6). Figs. 4-6 show the contribution of each variable to variance of the maximum principal stress at crown, the minimum principal stress at spring line and the maximum shear stress at crown, respectively. For the maximum principal stress at crown and the minimum principal stress at spring line, the elastic modulus has a quite large effect until 10,000 years and Poisson's ratio also has an effect. This is because the change in the results for the increment of the variables is relatively large as shown in Tables $\mathbf{5}$ and $\mathbf{6}$ and the standard deviation is also large in comparison with the increment. The results mean that the variation of elastic properties gives the large difference in the maximum and the minimum principal stresses during the occurrence of thermal stress. On the other hand, for the maximum shear stress at crown, the radial and horizontal stresses have a large effect. The elastic modulus becomes influential after 100,000 years. Since the shear stress is not related to the volumetric change, the thermal stress does not have an effect on the shear behavior. It is, however, noticeable that the contribution to the variance of the maximum shear stress is very small in comparison with that to the 
maximum and the minimum principal stresses.

Fig. 7 shows the reliability index of each stress. The reliability index of maximum shear stress is large until 1,000 years after closing the repository and then becomes steady value from 1 to 2 . That of the maximum principal stress becomes large gradually and the highest reliability is observed after one million years. The reliability index of the minimum

Table 4 Parameters at EDZ used for examination

\begin{tabular}{lcccc}
\hline $\begin{array}{l}\text { Distance } \\
\text { from } \\
\text { tunnel } \\
\text { wall }(\mathrm{m})\end{array}$ & $\begin{array}{l}\text { Fracture } \\
\text { density } \\
\left(\text { Num. } / \mathrm{m}^{2}\right)\end{array}$ & $\begin{array}{l}\text { Average } \\
\text { fracture } \\
\text { length } \\
L(\mathrm{~m})\end{array}$ & $\begin{array}{l}\text { Initial } \\
\text { damage } \\
\text { variable } \\
D_{0}\end{array}$ & $\begin{array}{l}\text { Initial } \\
\text { damage } \\
\text { potential } \\
B_{0}(\mathrm{kPa})\end{array}$ \\
\hline $0-0.2$ & 35 & 0.3 & 0.3 & 50 \\
$0.2-0.3$ & 25 & 0.3 & 0.1 & 60 \\
$0.3-0.4$ & 15 & 0.3 & 0.1 & 65 \\
$0.4-$ & 5 & 0.3 & 0.0 & 80 \\
\hline
\end{tabular}

Table 5 Maximum principal stress at crown of elastic case

\begin{tabular}{cccccc}
\hline Years & 100 & 1000 & 10000 & 100000 & 1000000 \\
\hline Variable & \multicolumn{5}{c}{ Maximum principal stress (MPa) } \\
\hline Mean & -42.0 & -56.7 & -69.5 & -76.1 & -76.4 \\
$\sigma_{v}$ & -40.5 & -55.1 & -67.9 & -74.5 & -74.9 \\
$\sigma_{h}$ & -45.7 & -60.4 & -73.2 & -79.8 & -80.1 \\
$\sigma_{r}$ & -42.0 & -56.6 & -69.4 & -76.1 & -76.4 \\
$P$ & -41.6 & -56.4 & -69.3 & -76.1 & -76.4 \\
$T$ & -42.0 & -56.7 & -69.5 & -76.1 & -76.4 \\
$E$ & -37.6 & -51.8 & -66.6 & -75.7 & -76.4 \\
$v$ & -35.3 & -50.1 & -65.9 & -75.6 & -76.4 \\
\hline
\end{tabular}

Table 6 Minimum principal stress at spring line of elastic case

\begin{tabular}{cccccc}
\hline Years & 100 & 1000 & 10000 & 100000 & 1000000 \\
\hline Variable & \multicolumn{5}{c}{ Minimum principal stress (MPa) } \\
\hline Mean & 67.3 & 47.9 & 9.39 & -14.0 & -14.9 \\
$\sigma_{v}$ & 63.6 & 44.3 & 5.78 & -17.6 & -18.5 \\
$\sigma_{h}$ & 68.8 & 49.5 & 11.0 & -12.4 & -13.3 \\
$\sigma_{r}$ & 67.3 & 47.9 & 9.41 & -14.0 & -14.8 \\
$P$ & 67.6 & 48.1 & 9.50 & -14.0 & -14.9 \\
$T$ & 67.3 & 47.9 & 9.39 & -14.0 & -4.9 \\
$E$ & 71.6 & 52.7 & 12.3 & -13.6 & -14.8 \\
$v$ & 74.0 & 54.5 & 13.0 & -13.5 & -14.8 \\
\hline
\end{tabular}

Table 7 Maximum shear stress at crown of elastic case

\begin{tabular}{cccccc}
\hline Years & 100 & 1000 & 10000 & 100000 & 1000000 \\
\hline Variable & \multicolumn{5}{c}{ Maximum shear stress $(\mathrm{MPa})$} \\
\hline Mean & 43.7 & 40.6 & 31.0 & 24.8 & 24.7 \\
$\sigma_{v}$ & 43.0 & 39.8 & 30.2 & 24.1 & 24.0 \\
$\sigma_{h}$ & 45.6 & 42.3 & 32.8 & 26.7 & 26.4 \\
$\sigma_{r}$ & 43.7 & 40.5 & 30.9 & 24.8 & 24.7 \\
$P$ & 43.7 & 40.6 & 31.0 & 24.8 & 24.7 \\
$T$ & 43.7 & 40.6 & 31.0 & 24.8 & 24.7 \\
$E$ & 43.7 & 40.6 & 31.0 & 24.9 & 24.7 \\
$v$ & 43.7 & 40.5 & 31.0 & 24.8 & 24.7 \\
\hline
\end{tabular}

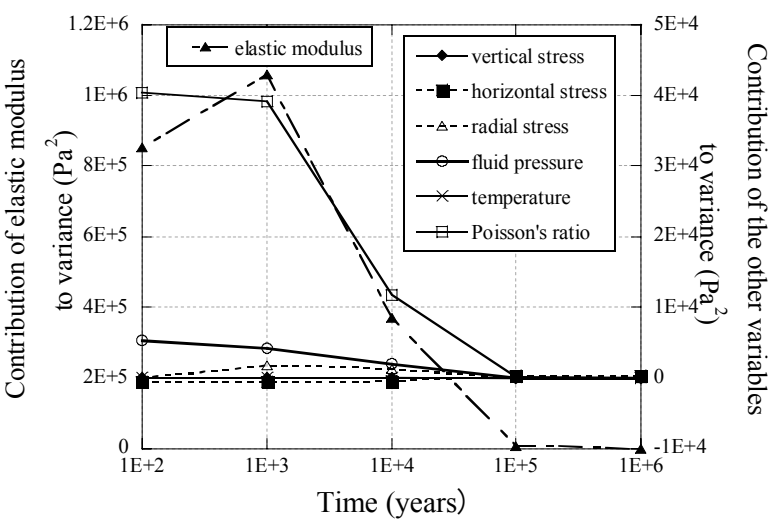

Fig.4 Contribution to variance of each variable for the maximum principal stress in elastic case. The left vertical axis is for the elastic modulus and the right one is for the other variables.

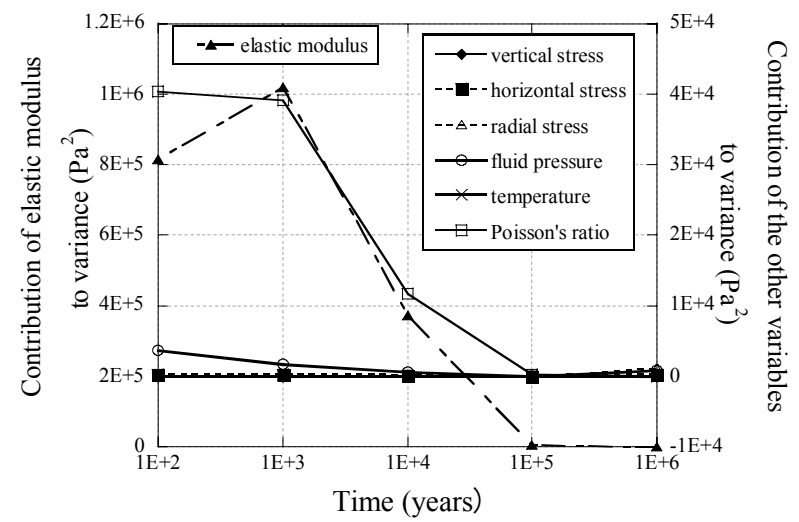

Fig.5 Contribution to variance of each variable for the minimum principal stress in elastic case. The left vertical axis is for the elastic modulus and the right one is for the other variables.

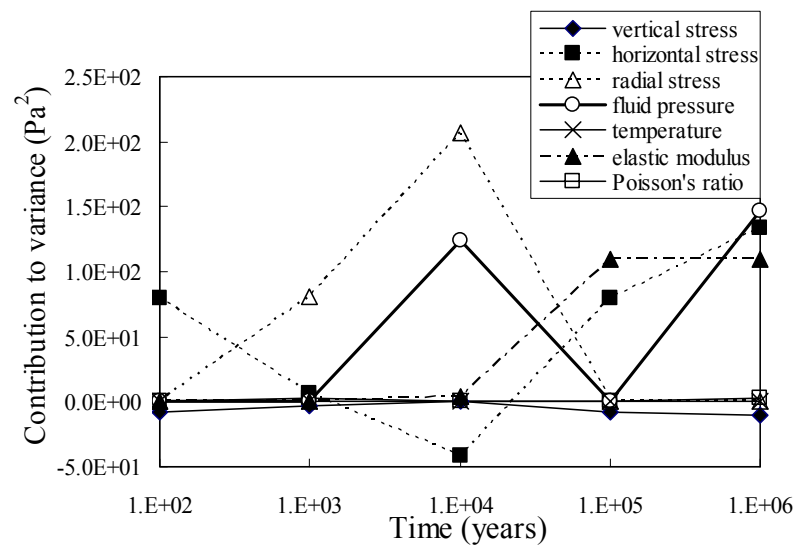

Fig.6 Contribution to variance of each variable for the maximum shear stress in elastic case 


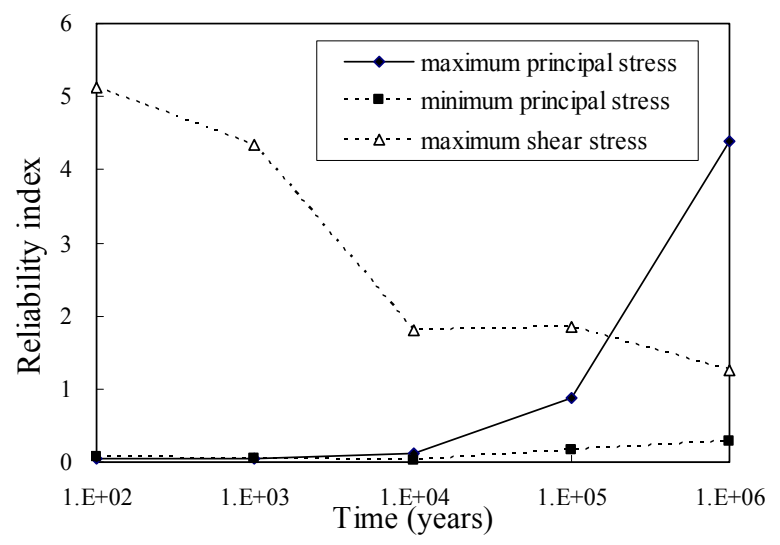

Fig.7 Reliability index in elastic case

principal stress is small through one million years.

It is found from the above results that the uncertainty of the predicted maximum shear stress is low through the evaluation period, while that of the minimum principal stress is high through the period and that of maximum principal stress changes. The high uncertainty of the minimum and maximum principal stresses until 10,000 years is related to the occurrence of thermal stress.

\section{(2) Damage model}

Tables 8-10 show the maximum principal stress at crown, the minimum principal stress at spring line and the maximum shear stress at crown of damage model case. In comparison with the results of elastic model, the stress becomes small entirely while the tendency of temporal change is similar. This is because the rock becomes soft by damage development. From the results, the contribution to variance of each variable is obtained as shown in Figs. 8-10. For the maximum principal stress, the elastic modulus and Poisson's ratio has a large effect until 10,000 years after closing the repository. The horizontal stress has a mostly constant effect throughout one million years. For the minimum principal stress, Poisson's ratio has a very large influence after 1,000 years. The elastic modulus has a similar contribution to the maximum principal stress. For the maximum shear stress, while the effect of each variable is the mostly same throughout one million years, horizontal stress has a relatively large effect. These tendencies are quite different from those of elastic case. However, the contribution is relatively small in comparison with that of elastic case. The large effect of Poisson's ratio on the minimum principal stress is caused from the expansive strain including the volumetric change due to damage progress. The reason why the horizontal stress has an effect is that the deformation is influenced by the horizontal stress. It is found that the contribution to the uncertainty is quite dependent on
Table 8 Maximum principal stress at crown of damage model case

\begin{tabular}{cccccc}
\hline Years & 100 & 1000 & 10000 & 100000 & 1000000 \\
\hline Variable & \multicolumn{5}{c}{ Maximum principal stress (MPa) } \\
\hline Mean & -28.8 & -40.8 & -51.5 & -57.1 & -57.4 \\
$\sigma_{v}$ & -27.8 & -39.8 & -505 & -56.1 & -56.4 \\
$\sigma_{h}$ & -31.3 & -43.3 & -54.0 & -59.6 & -59.9 \\
$\sigma_{r}$ & -28.8 & -40.8 & -51.5 & -57.1 & -57.4 \\
$P$ & -28.8 & -40.8 & -51.5 & 57.1 & -57.4 \\
$T$ & -28.8 & -40.8 & -51.5 & -57.1 & -57.4 \\
$E$ & -28.7 & -40.8 & -51.5 & -57.1 & -57.4 \\
$V$ & -28.4 & -40.6 & -51.4 & -57.1 & -57.4 \\
\hline
\end{tabular}

Table 9 Minimum principal stress at spring line of damage model case

\begin{tabular}{cccccc}
\hline Years & 100 & 1000 & 10000 & 100000 & 1000000 \\
\hline Variable & \multicolumn{5}{c}{ Minimum principal stress (MPa) } \\
\hline Mean & 52.9 & 36.9 & 5.54 & -13.5 & -14.2 \\
$\sigma_{v}$ & 52.0 & 34.4 & 3.01 & -16.1 & -16.8 \\
$\sigma_{h}$ & 52.7 & 36.3 & 5.56 & -13.5 & -14.2 \\
$\sigma_{r}$ & 52.9 & 37.0 & 5.56 & -13.5 & -14.2 \\
$P$ & 52.9 & 37.0 & 5.55 & -13.5 & -14.2 \\
$T$ & 52.9 & 36.9 & 5.54 & -13.5 & -14.2 \\
$E$ & 53.0 & 37.0 & 5.57 & -13.5 & -14.2 \\
$v$ & 53.0 & 35.6 & 4.05 & -15.1 & -15.8 \\
\hline
\end{tabular}

Table 10 Maximum shear stress at crown of damage model case

\begin{tabular}{cccccc}
\hline Years & 100 & 1000 & 10000 & 100000 & 1000000 \\
\hline Variable & \multicolumn{5}{c}{ Maximum shear stress (MPa) } \\
\hline Mean & 37.3 & 32.8 & 22.2 & 15.6 & 15.4 \\
$\sigma_{v}$ & 36.8 & 32.4 & 21.8 & 15.1 & 14.9 \\
$\sigma_{h}$ & 38.6 & 34.1 & 23.5 & 16.9 & 16.7 \\
$\sigma_{r}$ & 37.3 & 32.8 & 22.2 & 15.6 & 15.4 \\
$P$ & 37.3 & 32.8 & 22.2 & 15.6 & 15.4 \\
$T$ & 37.3 & 32.8 & 22.2 & 15.6 & 15.4 \\
$E$ & 37.3 & 32.8 & 22.2 & 15.6 & 15.4 \\
$V$ & 37.3 & 32.8 & 22.2 & 15.6 & 15.4 \\
\hline
\end{tabular}

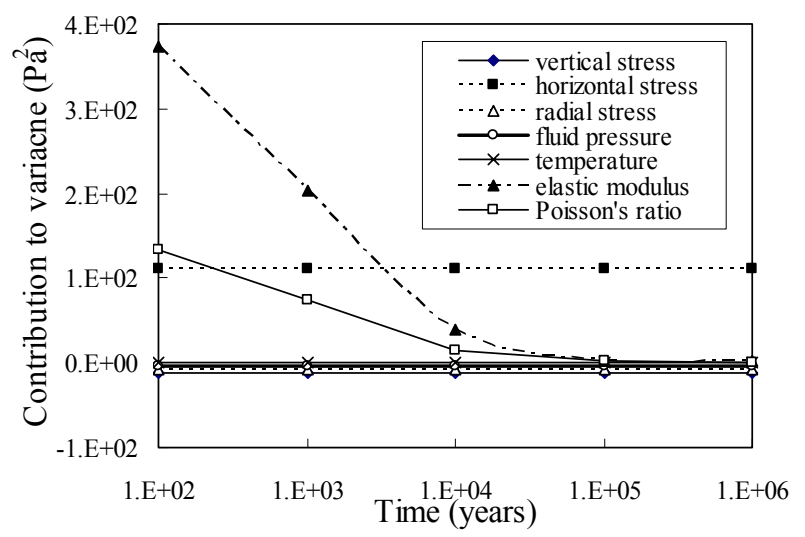

Fig.8 Contribution to variance of each variable for the maximum principal stress in damage model case 


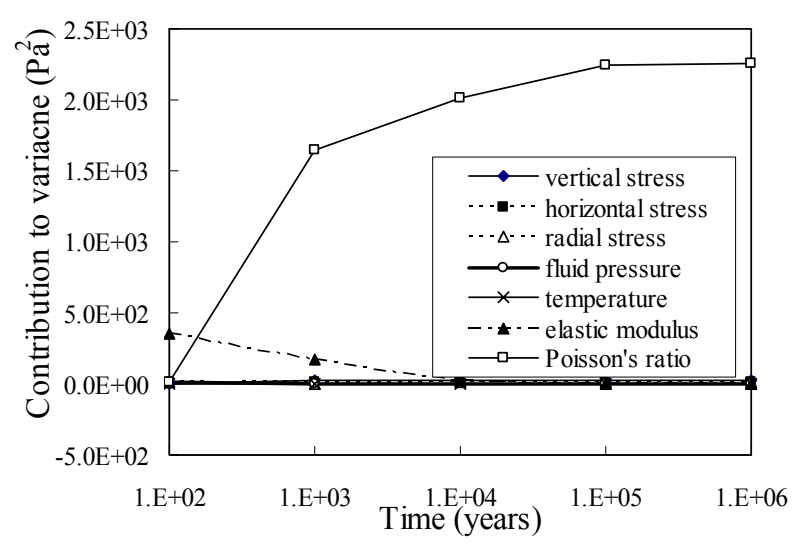

Fig.9 Contribution to variance of each variable for the minimum principal stress in damage model case

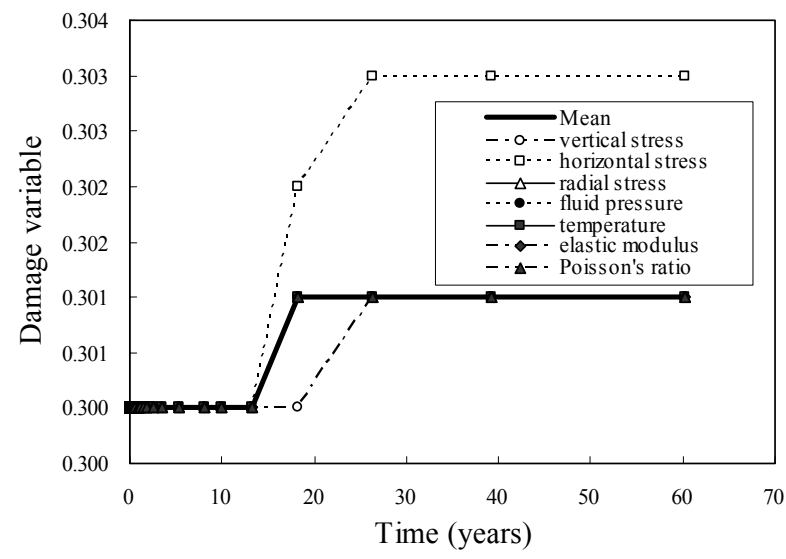

Fig.11 Damage variable evolution

the mechanical model.

Figs. 11 and 12 show the temporal variation of the damage variable and permeability at crown. The variation occurs after 18 and 26 years at which temperature becomes about peak value. The permeability is more responsive to the damage development than damage variable. When horizontal stress is incremented, the development of damage becomes large and permeability is changed drastically. After that, the damage variable and permeability do not change until the end of calculation. This means that maximum elastic work occurs at the time when temperature becomes peak.

Fig. 13 shows the evolution of reliability index of each stress. The reliability index of maximum principal stress gradually increases while that of the maximum shear stress decreases. That of the minimum principal stress is relatively small throughout a long period. This tendency is similar to that of elastic case while the value of reliability index of each stress is a little large in the damage model.

Table 11 shows the contribution of the variable to

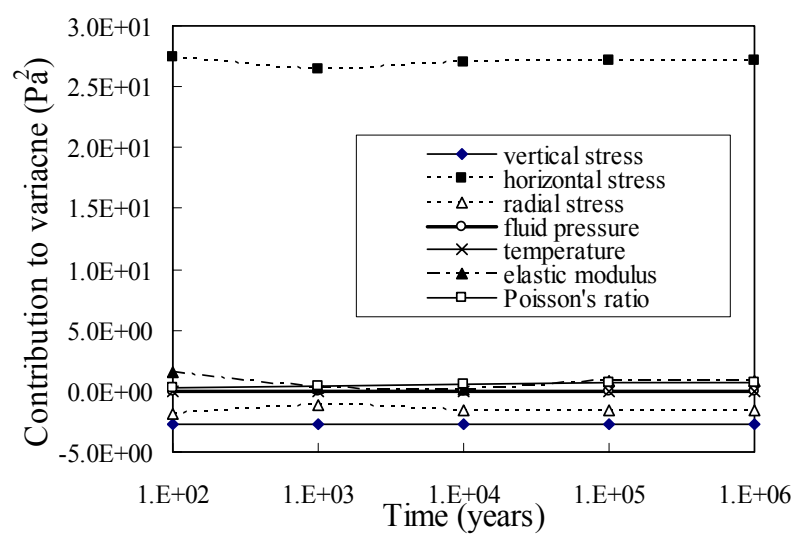

Fig.10 Contribution to variance of each variable for the maximum shear stress in damage model case

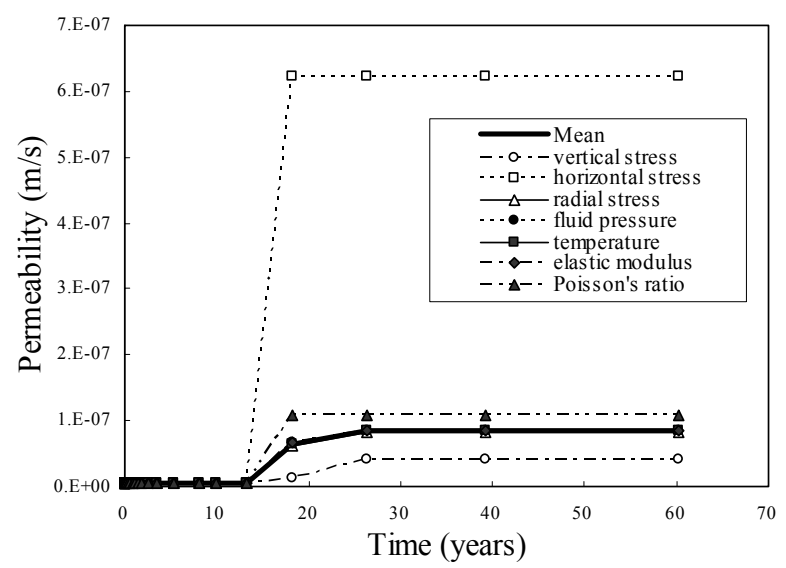

Fig. 12 Permeability evolution

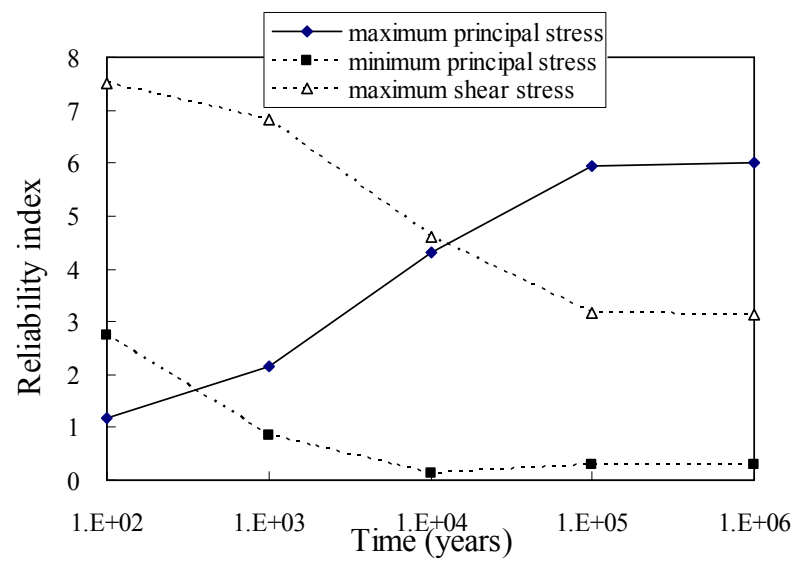

Fig.13 Reliability index of maximum principal stress, minimum principal stress and maximum shear stress of damage model case

the variance of damage variable and permeability, and the reliability indexes at 100 years. These values do not vary after 100 years. In the table, the reliability indexes for both damage variable and permeability are also indicated, which are also the values remained 
Table 11 Contribution of each variable to variance and reliability index for damage variable $D$ and permeability $K(\mathrm{~m} / \mathrm{s})$ at 100 years

\begin{tabular}{cccccc}
\hline & Mean & $\sigma_{v}$ & $\sigma_{h}$ & $\sigma_{r}$ & $P$ \\
\hline$D$ & 0.301 & $-3.5 \mathrm{E}-6$ & $2.9 \mathrm{E}-5$ & $5.7 \mathrm{E}-6$ & $-1.7 \mathrm{E}-6$ \\
$K$ & $8.4 \mathrm{E}-8$ & $-1.5 \mathrm{E}-13$ & $6.9 \mathrm{E}-12$ & $-7.7 \mathrm{E}-13$ & $1.3 \mathrm{E}-13$ \\
\hline & $T$ & $E$ & $v$ & $\beta$ & \\
\hline$D$ & 0 & $1.8 \mathrm{E}-5$ & $5.6 \mathrm{E}-5$ & 29.7 & \\
$K$ & 0 & $1.0 \mathrm{E}-13$ & $5.5 \mathrm{E}-13$ & 0.032 & \\
\hline
\end{tabular}

after 100 years. The contribution of horizontal stress is larger than the other variables for both damage variable and permeability. The reliability of the damage variable is high and that of permeability is quite low.

\section{CONCLUSIONS}

In this paper, the uncertainty analysis of the mechanical behavior of near field of HLW repository was examined. The uncertainty of the maximum principal stress at crown of the repository tunnel, the minimum principal stress at spring line and maximum shear stress at crown were examined for the variables of boundary conditions, elastic modulus and Poisson's ratio. The variance of boundary conditions was obtained from the temporal variation for one million years which were estimated from the far field analysis.

To examine the contribution of the variables to the variance of each stress, the first order second moment method was applied. As a result, the followings were obtained:

1) For the maximum principal stress at crown, the elastic modulus and Poisson's ratio had a large effect on the variance in the elastic and the damage expansion models.

2) For the minimum principal stress at spring line, the elastic modulus had an effect on the variance in both models. In the damage expansion model, the Poisson's ratio had a large influence.

3) For the maximum shear stress at crown, the boundary condition had a larger effect than the elastic properties in both models.

4) The reliability index of maximum stress gradually decreased, that of maximum principal stress gradually increased and that of minimum principal stress was small through a long period. This tendency was similar for both models.

5) For damage variable and permeability at crown, the horizontal stress on the boundary had a large influence.

For the elastic case, the occurrence of the thermal stress had an effect on the uncertainty of the behavior around the tunnel. On the other hand, the damage expansion model showed the effect of mechanicalwork on the uncertainty. The uncertainty of the prediction is dependent on the mechanical model. It was, however, found from above results that estimation of boundary conditions was relatively important to examine the stability and permeability change around the repository tunnel as well as the examination of uncertainty of mechanical properties. Besides, since the reliability of the minimum principal stress was expected to be low, careful consideration is needed for the prediction of tension failure and expansion of crack aperture at the spring line.

The effect of temperature could not be identified for the near field model from the method used in this paper. For this purpose, the boundary conditions describing temperature condition has to be applied.

\section{REFERENCES}

1) Japan Nuclear Cycle Development Institute: H12 : Project to Establish the Scientific and Technical Basis for HLW Disposal in Japan, 1999.

2) The Research Group of Active Faults of Japan: Active Faults of Japan -their distribution and characteristics-, Univ. Tokyo Press, p.437, 1991 (in Japanese).

3) Research Group for Quaternary Tectonic Maps: Quaternary Tectonic Map of Japan, Dai-Yonki-Kenkyuu (Reports for the Quaternary), 7, pp.182-187, 1968 (in Japanese).

4) Emiliani, C.: The cause of the ice ages, Earth and Planetary Science Letters, 37, pp. 349-352, 1978.

5) Swedish Nuclear Power Inspectorate: DECOVALEX-THMC Project, TaskB, Understanding and characterizing the excavation disturbed zone (EDZ), Phase 2 Report, SKI Report 2007:08, 2007.

6) Baecher, G.B., and Christian, J.T.: Reliability and statistics in geotechnical engineering, Wiley, pp.325-327, 2003.

7) Gomez-Hernandez, J. J. and Cassiraga, E. F.: Impact of flow and transport coupling in the upscaling of transport parameters for performance assessment in the context of nuclear waste disposal, $1^{\text {st }}$ International conference on coupled thermo - hydro - mechanical - chemical processes in geosystems and engineering, pp.233-238, 2003.

8) Long, J. C. S., Remer, J. S., Wilson, C. R. and Witherspoon, P. A.: Porous media equivalents for networks of discontinuous fractures, Water Resources Research, Vol. 18, No. 3, pp.645 658, 1982.

9) Rutqvist, J., Bilkholzer, J. T., Chijimatsu, M., Kolditz, O., Quansheng Lij, Oda, Y. and Chengyuan, Z.: Comparative simulation study of coupled THM processes near back-field and open-drift nuclear waste repositories in Task D of the international DECOVALEX project, 2nd International conference on coupled thermo - hydro - mechanical - chemical processes in geosystems and engineering, pp. 428-433, 2006.

10) Lee, I.K., White, W. and Ingels, O. G.: Geotechnical Engineering, Boston, Pitman, 1983.

11) Rosenblueth, E. : Point estimates for probability moments, Proceedings, National Academy of Science, Vol. 72, No. 10, pp. 3812-3814, 1975.

12) Hasofer, A.M. and Lind, N.C.: An exact and invariant first-order reliability format, Journal of the Engineering Mechanics Division, ASCE 100(EM1), pp.111-121, 1974.

13) Christian, J.T., Ladd, C.C. and Baecher, G.B.: Reliability 
applied to slope stability analysis, Journal of Geotechnical Engineering, ASCE 120(2), pp.2180-2207, 1994.

14) Rutqvist, J., Feng, X-T., Hudson, J., Jing, L., Kobayashi, A., Koyama, T., Pan, P-Z., Lee, H-S., Rinne, M., Sonnenthal, E. and Yamamoto, K.: Multi-code benchmark simulation study on coupled THMC process in the excavation disturbed zone associated with geological nuclear waste repositories, 2nd International conference on coupled thermo - hydro - mechanical - chemical processes in geosystems and engineering, pp. 397-402, 2006.

15) Lemaitre, J.: A course on damage mechanics, Berlin, Springer-Verlag, 1992.

16) Yamamoto, K., Kobayashi, A. and Aoyama, S.: Numerical analysis with damage mechanics for degraded rocks, International Conference on Computational \& Experimental Engineering and Sciences, pp.1973-1978, 2005.

17) Murakami, S. and Kamiya, K.: Constitutive and damage evolution equations of elastic-brittle materials based on irreversible thermodynamics, International Journal of Mechanical Sciences, Vol. 39, No. 4, pp.473-486, 1997.
18) Yamamoto, K., Kobayashi, A. and Aoyama, S.:Change in damage parameters of rocks due to degradation, International Workshop on Prediction and Simulation Methods in Geomechanics, pp. 49-52, 2003.

19) Bäckström, A., Lanaro, F. and Christiansson, R.: Coupled chemical-mechanical behavior: The influence of salinity on the uniaxial compressive strength of the Smålands granite, Sweden, 2nd International conference on coupled thermo hydro - mechanical - chemical processes in geosystems and engineering, pp. 437-443, 2006.

20) Kobayashi, A., Yamamoto, K., Aoyama, S., Chijimatsu, M. and Fujita, T.: Changes in intact rock damage parameters due to chemical effects and their influence on failure phenomena, 2nd International conference on coupled thermo - hydro mechanical - chemical processes in geosystems and engineering, pp. 281-286, 2006.

(Received December 4, 2007) 